\title{
Gender sensitive education program in eastern Jerusalem Arab schools
}

\author{
Diana Daaboul -Phd.
}

\begin{abstract}
In recent years, the Gender and Gender Equality Unit has been launched, and a number of enrichment and enrichment programs have been launched within schools, students participating in empowerment workshops, special programs and educational initiatives, both on behalf of the Ministry of Education in israel and such Belonging to external programs.

This process, which is growing in the non-Arab educational system in Israel, is parallel to a similar process in the Arab education system or in Arab society in Israel, but more slowly, less holistic and assimilated and less continuous. This is despite the need for such programs and interventions in the Arab society in Israel, in all its layers (more and less traditional).

In our rapidly changing world there are still issues that are always discussed in one way or another, they are becoming more complex or are not fully resolved, and it is always possible to develop and propose programs and initiatives and studies that deal with them. Gender and gender equality are part of these issues.

Traditional societies in general and Arab society in Israel in particular live in a rift between tradition and modernity. On the one hand, children live in a traditional society with restrictive social characteristics in many areas of life. On the other hand they are exposed to a wide Western culture, the gender issue remains relevant today, despite and in conjunction with a number of programs and initiatives on this subject in Arab society in Israel and in Arab society in general.
\end{abstract}


In addition, children and girls (in Arab society and other societies) are also exposed to a wide technological world, receiving messages and materials that influence their personality, personal and social development, and directly on their scholastic achievements. And here, parents are not always a source of inspiration, because the other channels are open and accessible.

Sexual harassment is a social phenomenon in children's lives and is usually caused by crossing the gender boundary. Crossing this border is an expression of power in the children's society and usually occurs far from adults. Sexual harassment is influenced by behavioral norms in society and by children's exposure to sexual stereotypes in the media and on the Internet. In such a reality, the child needs significant tools to search for and find their "personal selves," empowering the personal, emotional and social awareness, to look within themselves and to recognize their skills and to know what their limits are and what are the boundaries of the other. They also need tools that increase their awareness of the "other" whether they are a boy or a girl, and empower their ability to communicate with them positively and positively. To liberate stereotypical views, and to educate according to the values of honor and equal opportunity for both sexes. And to develop critical thinking regarding stereotypes, values, and behaviors among students, teachers and parents alike.

And especially girls, the gifted daughter is at risk of being in conflict between her desires and the desires of the society to which she belongs, between her aspirations for academic and professional progress, and confusing messages (which is true of some of the boys).

Alienation, lack of familiarity, prejudices, inappropriate treatment, and poor communication can break up companies. Investing in the design of a person who is sensitive to himself and his gender, and a sensitive person to the complementary part, through a formative education system that intensifies gender, will lead to the creation of a sensitive society for all the people who make it a strong and protected society. 
Children who receive tools of the Gender Sensitive Education Program, which will serve as a basis for personal empowerment in the gender / personal sphere, and a basis for proper and sensitive knowledge of gender in traditional society and a basis for positive communication between the sexes, They will be able to realize themselves, to defend themselves, to find the path and learning style that suits them best, and to be adults who can better cope in a multicultural society in the 21st century. They will grow and grow and become graduates of the Arab society who can lead it and to live within it in a practical manner, according to worldviews and egalitarian culture.

At this conference I will define my gender-sensitive education program that is an evolving program that believes that the education system really influences the design of gender perceptions of students and students. It will operate in three main arenas:

The school arena: division of labor and organizational culture, adaptation of the curriculum and the learning environment. Creating a school commitment to gender equality. And it is structured with the intention that the school accepts full gender responsibility to change the administrative conditions and pedagogies so that they reflect loyalty to gender equality.

The application is comprehensive and holistic, and includes all areas of school activity and includes parents, teachers, management and students alike.

This program is the first of its kind and is unique in schools in East Jerusalem. It was designed after much research and work. It is still developing and accompanied by evaluation research. The program is suitable for educators and other heterogeneous and traditional educational systems, and its presentation will contribute to its expansion and its more efficient application 